\title{
On a parametric family of Thue inequalities over function fields
}

\section{Journal Article}

Author(s):

Fuchs, Clemens; Jadrijevic, Borka

Publication date:

2007

Permanent link:

https://doi.org/10.3929/ethz-b-000006114

Rights / license:

In Copyright - Non-Commercial Use Permitted

Originally published in:

Mathematical Proceedings of the Cambridge Philosophical Society 143(1), https://doi.org/10.1017/S0305004107000217 


\title{
On a parametric family of Thue inequalities over function fields
}

\author{
By CLEMENS FUCHS \\ ETH Zurich, Department of Mathematics, Rämistrasse 101, 8092 Zürich, Switzerland. \\ e-mail: clemens.fuchs@math.ethz.ch \\ AND BORKA JADRIJEVIĆ \\ University of Split, FESB, R. Boškovića bb, 21000 Split, Croatia. \\ University of Split, Department of Mathematics, Teslina 12, 21000 Split, Croatia. \\ e-mail: borka@pmfst.hr
}

(Received 16 February 2006; revised 30 May 2006)

\begin{abstract}
In this paper we completely solve a family of Thue inequalities defined over the field of functions $\mathbb{C}(T)$, namely $\operatorname{deg}\left(X^{4}-4 c X^{3} Y+(6 c+2) X^{2} Y^{2}+4 c X Y^{3}+Y^{4}\right) \leqslant \operatorname{deg} c$, where the solutions $x, y$ come from the ring $\mathbb{C}[T]$ and the parameter $c \in \mathbb{C}[T]$ is some non-constant polynomial.
\end{abstract}

\section{Introduction}

Let $F \in R[X, Y]$ be a binary irreducible form of degree $d \geqslant 3$ over some ring $R$. An equation of the form

$$
F(X, Y)=m, \quad m \in R^{\times}
$$

is called a Thue equation, due to Thue [22] who proved, in the case $R=\mathbb{Z}$, that such an equation has finitely many solutions. In the last decade, starting with the result of Thomas in [21], several families (at the moment up to degree 8; see [9] and the references mentioned therein) of Thue equations have been considered, where the coefficients of the form $F_{c}(X, Y)$ depend on an integral parameter $c$ in $R=\mathbb{Z}$ or the integral closure of $\mathbb{Z}$ in an imaginary quadratic number field.

It is due to Gill [8] and later Schmidt [19] and Mason $[\mathbf{1 4}, \mathbf{1 5}]$ that single Thue equations can also be solved effectively over function fields. Recently, Lettl gave some new insights in this subject (cf. $[\mathbf{1 1}, \mathbf{1 2}]$ ). However, no family of Thue equations over function fields was considered by these authors. Very recently, some families of Thue equations with parameter $c \in \mathbb{C}[T]$ were studied by Ziegler and the first author. These were the first such results over function fields. In [6] all solutions $(x, y) \in \mathbb{C}[T] \times \mathbb{C}[T]$ of the family

$$
X^{3}-(T+\xi+1) X^{2} Y+(T+\xi) X Y^{2}+Y^{3}=-\xi T^{2}+\xi T+1
$$

for all $\xi \in \mathbb{C}$ were found, namely $\left\{\zeta(T, 1): \zeta^{3}=1\right\}$ with some additional solutions for $\xi=-1,0,1$ (see [6]). Moreover, in [7], all solutions of the function field analogue of Thomas' family [21]

$$
X^{3}-(c-1) X^{2} Y-(c+2) X Y^{2}-Y^{3}=\xi
$$


Table 1. Solutions and their right-hand sides

\begin{tabular}{cc}
\hline$m$ & $(x, y) \in \mathcal{L}$ \\
\hline$a^{4}$ & $(a, 0),(0, a)$ \\
$25 a^{4}$ & $(2 a, a),(a,-2 a)$ \\
\hline
\end{tabular}

where $c \in \mathbb{C}[T]$ is a non-constant polynomial and $\xi \in \mathbb{C}^{\times}:=\mathbb{C} \backslash\{0\}$, were calculated. We have that all solutions $(x, y) \in \mathbb{C}[T] \times \mathbb{C}[T]$ are contained in $\{(\zeta, 0),(-\zeta, \zeta),(0,-\zeta)$ : $\left.\zeta^{3}=\xi\right\}$. In the proof we essentially followed the ideas of Mason [14].

Apart from families of Thue equations also Thue inequalities

$$
|F(X, Y)| \leqslant m
$$

over $R=\mathbb{Z}$, where studied previously, but up to now only few parametrised inequalities of small degree have been solved (see [13, 17, 25-27]). We mention a result of Dujella and the second author (see [4]), who proved that the only primitive (i.e. with coprime components) solutions of the Thue inequality

$$
\left|X^{4}-4 c X^{3} Y+(6 c+2) X^{2} Y^{2}+4 c X Y^{3}+Y^{4}\right|<6 c+4
$$

where $c \geqslant 4$ is an integer, are $(x, y) \in\{( \pm 1,0),(0, \pm 1),(1, \pm 1),(-1, \pm 1),( \pm 1, \mp 2)$, $( \pm 2, \pm 1)\}$ (see also [3]). In [10] the second author, jointly with Ziegler, generalised this result by considering the analogue family of equations over imaginary quadratic number fields.

In this paper, we will consider a function field analogue of the family of Thue inequalities considered above, namely

$$
\operatorname{deg}\left(X^{4}-4 c X^{3} Y+(6 c+2) X^{2} Y^{2}+4 c X Y^{3}+Y^{4}\right) \leqslant \operatorname{deg} c,
$$

where solutions $x, y$ come from the ring $\mathbb{C}[T]$ and the parameter $c \in \mathbb{C}[T]$ is some nonconstant polynomial.

First observe that the problem is trivial for solutions $(x, y) \in \mathbb{C}[T] \times \mathbb{C}[T]$ with deg $x=$ $\operatorname{deg} y=0$, since all $(x, y) \in \mathbb{C}^{2}$ give solutions to the Thue inequality since in this case $x^{4}-4 c x^{3} y+(6 c+2) x^{2} y^{2}+4 c x y^{3}+y^{4}$ is a polynomial of degree $\leqslant \operatorname{deg} c$. Therefore we restrict to solutions $(x, y) \in(\mathbb{C}[T] \times \mathbb{C}[T]) \backslash \mathbb{C}^{2}$. Our main result is the following theorem.

THEOREM 1. The set of solutions $\mathcal{L} \subseteq(\mathbb{C}[T] \times \mathbb{C}[T]) \backslash \mathbb{C}^{2}$ of the family of Thue inequalities

$$
\operatorname{deg}\left(X^{4}-4 c X^{3} Y+(6 c+2) X^{2} Y^{2}+4 c X Y^{3}+Y^{4}\right) \leqslant \operatorname{deg} c,
$$

where $c \in \mathbb{C}[T]$ is a non-constant polynomial, is given by

$$
\mathcal{L}=\{(a, 0),(0, a),(2 a, a),(a,-2 a): a \in \mathbb{C}[T] \text { with } 0<4 \operatorname{deg} a \leqslant \operatorname{deg} c\} .
$$

Clearly, the Thue inequality $(1 \cdot 1)$ can be rewritten as

$$
X^{4}-4 c X^{3} Y+(6 c+2) X^{2} Y^{2}+4 c X Y^{3}+Y^{4}=m \text {, where } \operatorname{deg} m \leqslant \operatorname{deg} c .
$$

We collect the solutions of $(1 \cdot 1)$ from Theorem 1 according to the corresponding right-hand side $m$ of the equation (1.2) in the Table 1 (where as above $a \in \mathbb{C}[T]$ with $0<4 \operatorname{deg} a \leqslant$ $\operatorname{deg} c$ ).

The novelty of this result is threefold. Firstly it is the first parametrised family of Thue inequalities over function fields solved up to now. Secondly if we look at the corresponding 
families of Thue equations, which are solved with this family, then it is the family of the largest degree considered yet (up to now only cubic families were considered, whereas we have a quartic family). Last, but not least, the method of proof is completely different from the way the results in $[6,7]$ are obtained as we will describe below.

In order to attack the inequality we will use a reduction method, which has origins in the method of Tzanakis [24], and show that inequality $(2 \cdot 2)$ corresponds to systems of the form

$$
\begin{aligned}
& (2 c+1) U^{2}-2 c V^{2}=m, \\
& (c-2) U^{2}-c Z^{2}=-2 m .
\end{aligned}
$$

where $\operatorname{deg} m \leqslant \operatorname{deg} c$. This will be done in Section 2, where we will also give some information about the splitting field of the form associated with the Thue equation.

In the proof we will also use the fact that there are lots of analogues between $\mathbb{C}[T], \mathbb{C}(T)$, $\mathbb{C}\left(\left(T^{-1}\right)\right)$ and $\mathbb{Z}, \mathbb{Q}, \mathbb{R}$, respectively. Especially, one can consider continued fraction expansion of an element in $\mathbb{C}\left(\left(T^{-1}\right)\right)$. Namely, we have - as in classical theory - that each element in $\mathbb{C}\left(\left(T^{-1}\right)\right)$ has a unique continued fraction expansion and an expansion of an element is finite if and only if the element is in $\mathbb{C}(T)$. We will give an overview about the theory of continued fractions for algebraic functions and prove some auxiliary results in Section 3.

In Section 4 we will use the theory of continued fractions in order to determine all values of $m$ with $\operatorname{deg} m \leqslant \operatorname{deg} c$, for which system $(1.3 a)$ and $(1.3 b)$ has a solution. In particular, a characterisation in terms of continued fractions of $\alpha \in \mathbb{C}\left(\left(T^{-1}\right)\right)$ of all rational functions $P / Q$ satisfying the inequality

$$
\left|\alpha-\frac{P}{Q}\right|<\frac{1}{|Q|^{2}}
$$

(see Proposition 1 in Section 3) is used.

Then, in Section 5, we apply the congruence method, which was first used in this context in [5] (see also [2]), to show that system $(1.3 a)$ and (1.3b) has only trivial primitive (i.e. with coprime components) solutions $(U, V, Z)=( \pm \zeta, \pm \zeta, \pm \zeta)$ for some $\zeta \in \mathbb{C}^{\times}$(cf. Corollary 1).

Finally, the proof of the main theorem will be completed in Section 6.

\section{Preliminaries and reduction to a system of inequalities}

We consider the field of rational functions $\mathbb{C}(T)$ with the following valuation:

$$
v\left(\frac{P}{Q}\right)=\operatorname{deg} P-\operatorname{deg} Q, \quad P, Q \in \mathbb{C}[T], \quad Q \neq 0,
$$

where we put $v(0)=-\infty$. This valuation can be extended to a valuation on the field of formal Laurent series $\mathbb{C}\left(\left(T^{-1}\right)\right)$ by

$$
v\left(\sum_{i=n}^{\infty} a_{i} T^{-i}\right)=-n, \quad a_{n} \neq 0
$$

With

$$
|\alpha|=b^{v(\alpha)}
$$

where $b \geqslant 2$ is an integer and $\alpha \in \mathbb{C}\left(\left(T^{-1}\right)\right)$, we get a non-archimedian absolute value on $\mathbb{C}\left(\left(T^{-1}\right)\right)$. In this notation our inequality $(1 \cdot 1)$ has the form

$$
\left|X^{4}-4 c X^{3} Y+(6 c+2) X^{2} Y^{2}+4 c X Y^{3}+Y^{4}\right| \leqslant|c| .
$$


First we state some easy to prove facts about the underlying algebraic structure of the form $F(X, Y)=X^{4}-4 c X^{3} Y+(6 c+2) X^{2} Y^{2}+4 c X Y^{3}+Y^{4}$. We consider

$$
f(X)=F(X, 1)=X^{4}-4 c X^{3}+(6 c+2) X^{2}+4 c X+1 .
$$

The zeros of $f(X)=0$ are given by

$$
\begin{aligned}
& \alpha_{1}=c+\sqrt{c(c-2)}+\sqrt{c\left(c+\frac{1}{2}\right)}+\sqrt{(c-2)\left(c+\frac{1}{2}\right)}, \\
& \alpha_{2}=c-\sqrt{c(c-2)}+\sqrt{c\left(c+\frac{1}{2}\right)}-\sqrt{(c-2)\left(c+\frac{1}{2}\right)}, \\
& \alpha_{3}=c+\sqrt{c(c-2)}-\sqrt{c\left(c+\frac{1}{2}\right)}-\sqrt{(c-2)\left(c+\frac{1}{2}\right)}, \\
& \alpha_{4}=c-\sqrt{c(c-2)}-\sqrt{c\left(c+\frac{1}{2}\right)}+\sqrt{(c-2)\left(c+\frac{1}{2}\right)} .
\end{aligned}
$$

Therefore, the splitting field of $f$ is

$$
\mathbb{C}\left(T, \sqrt{c(c-2)}, \sqrt{c\left(c+\frac{1}{2}\right)}\right)=\mathbb{C}(T)\left(\alpha_{1}\right)=: L .
$$

The field extension $L / \mathbb{C}(T)$ is a Galois extension and the Galois group is isomorphic to $C_{2} \times C_{2}$. Moreover, by using the Hurwitz formula [23, theorem III.4.12, page 88] the genus $g_{L}$ of $L / \mathbb{C}(T)$ is bounded by $g_{L} \leqslant(9 / 2) \operatorname{deg} c-3$, since all places in $L$ that are ramified over finite places $v_{a}$ in the rational function field satisfy $2 c^{3}(a)-3 c^{2}(a)-2 c(a)=0$. From what we have collected up to now it is rather easy to prove, using the theorem of Mason mentioned above (cf. [15, chapter 1, lemma 2]), that every solution $(x, y) \in \mathbb{C}[T] \times \mathbb{C}[T]$ of our inequality (2.2) satisfies $\max \{\operatorname{deg} x, \operatorname{deg} y\} \leqslant(41 / 4) \operatorname{deg} c-3 / 4$.

As mentioned in the introduction we will use another method of proof to obtain our result. We consider Thue equation (1.2), that was

$$
X^{4}-4 c X^{3} Y+(6 c+2) X^{2} Y^{2}+4 c X Y^{3}+Y^{4}=m,
$$

where solutions $x, y$ come from the ring $\mathbb{C}[T]$, parameter $c$ is some non-constant polynomial and $m \in \mathbb{C}[T]$ where $|m| \leqslant|c|$. We want to show how the solutions of the family of Thue equations $(1 \cdot 2)$ over the function field $\mathbb{C}(T)$ correspond to the solutions of the system $(1 \cdot 3 a)$ and $(1 \cdot 3 b)$ of Pellian equations over the same function field.

It is clear that every solution $(x, y) \in \mathbb{C}[T] \times \mathbb{C}[T]$ of Thue equation (1.2) yields a solution of the system

$$
\begin{aligned}
(2 c+1) U^{2}-2 c V^{2} & =m, \\
(c-2) U^{2}-c Z^{2} & =-2 m,
\end{aligned}
$$

where

$$
U=x^{2}+y^{2}, \quad V=x^{2}+x y-y^{2}, \quad Z=-x^{2}+4 x y+y^{2},
$$

because to show this we just have to insert $U, V, Z$ in the system of Pellian equations and we see that both equations reduce to the original Thue equation. In fact this can be done by the same computations as in [4], since these computations are still valid in $\mathbb{C}[T]$. Therefore, $\mathcal{L}$ (by the transformation $(2 \cdot 3))$ is contained in the set of solutions of $(1 \cdot 3 a)$ and $(1 \cdot 3 b)$. Conversely, if $U, V, Z$ is a solution of the system of Pellian equations we have to find all 
solutions of the system $(2 \cdot 3)$, to get all possible corresponding solutions of the Thue equation.

We mention that this relationship was also used by Dujella and Jadrijević in [4] and that there it was found by using the method of Tzanakis [24].

\section{Continued fractions}

In this section we will recall the theory of continued fraction expansion for algebraic functions in $\mathbb{C}(T)$. First of all, we will start with definitions and some notation. Everything here holds also over an arbitrary field instead of the field of complex numbers $\mathbb{C}$. For details see [16] and [20].

We define rational functions in polynomials $A_{0}, A_{1}, \ldots \in \mathbb{C}[T]$ by

$$
\begin{aligned}
{\left[A_{0}\right] } & =A_{0}, \quad\left[A_{0}, A_{1}\right]=A_{0}+\frac{1}{A_{1}}, \\
{\left[A_{0}, A_{1}, \ldots, A_{n}\right] } & =\left[A_{0}, A_{1}, \ldots, A_{n-2}, A_{n-1}+1 / A_{n}\right], \quad \text { for } n \geqslant 2,
\end{aligned}
$$

then

$$
\left[A_{0}, A_{1}, \ldots, A_{n}\right]=\left[A_{0}, A_{1}, \ldots, A_{k-1},\left[A_{k}, \ldots, A_{n}\right]\right] .
$$

Setting $P_{-2}=0, Q_{-2}=1, P_{-1}=1, Q_{-1}=0$,

$$
P_{n}=A_{n} P_{n-1}+P_{n-2}, \quad Q_{n}=A_{n} Q_{n-1}+Q_{n-2}, \quad n \geqslant 0,
$$

we observe that $P_{n}, Q_{n}$ are polynomials, that

$$
Q_{n} P_{n-1}-P_{n} Q_{n-1}=(-1)^{n}, \quad n \geqslant-1,
$$

and

$$
\left[A_{0}, A_{1}, \ldots, A_{n}\right]=\frac{P_{n}}{Q_{n}} .
$$

An element of $\mathbb{C}(T)$ may be uniquely expressed as $\left[A_{0}, A_{1}, \ldots, A_{n}\right]=P_{n} / Q_{n}$, where each $A_{i} \in \mathbb{C}[T]$ and where $\operatorname{deg} A_{i} \geqslant 1$ for $i \geqslant 1$. By (3.1), the polynomials $P_{n}, Q_{n} \in \mathbb{C}[T]$ are relatively prime, i.e. $\left(P_{n}, Q_{n}\right)=1$. Note that the pair $P_{n}, Q_{n}$ is determined by (3.2) and is relatively prime only up to a common factor in $\mathbb{C}^{\times}$, i.e. $\zeta P_{n}, \zeta Q_{n}$ with $\zeta \in \mathbb{C}^{\times}$ have the same properties. When $A_{0}, A_{1}, \ldots \in \mathbb{C}[T]$ are given with $\operatorname{deg} A_{i} \geqslant 1$ for $i \geqslant 1$, then $\left[A_{0}, A_{1}, \ldots, A_{n}\right]$ as $n \rightarrow \infty$ converges with respect to $|\cdot|$ the norm associated to the non-archimedean absolute value on $\mathbb{C}\left(\left(T^{-1}\right)\right.$ ) (as given by $\left.(2 \cdot 1)\right)$, to an element of $\mathbb{C}\left(\left(T^{-1}\right)\right.$ ) which will be denoted by

$$
\left[A_{0}, A_{1}, A_{2}, \ldots\right]=A_{0}+\frac{1}{A_{1}+\frac{1}{A_{2}+\frac{1}{\ddots}}} .
$$

Then we have that: every element $\alpha \in \mathbb{C}\left(\left(T^{-1}\right)\right) \backslash \mathbb{C}(T)$ can be uniquely expressed as such an infinite continued fraction. Moreover, precisely the elements from $\mathbb{C}(T)$ have a finite expansion.

Writing $\alpha=\left[A_{0}, A_{1}, \ldots\right]$, we call $P_{n} / Q_{n}$ as given by (3.2) the $n$th convergent, $A_{n}$ the $n$th partial quotient and $\alpha_{n}=\left[A_{n}, A_{n+1}, \ldots\right]$ the $n$th complete quotient. We have

$$
\alpha=\left[A_{0}, A_{1}, \ldots, A_{n-1}, \alpha_{n}\right]=\frac{\alpha_{n} P_{n-1}+P_{n-2}}{\alpha_{n} Q_{n-1}+Q_{n-2}}, \quad n \geqslant 1 .
$$


Legendre's theorem is also valid for this continued fraction expansion of elements of $\mathbb{C}\left(\left(T^{-1}\right)\right)$, namely we have:

Proposition 1. Let $\alpha$ be an element in $\mathbb{C}\left(\left(T^{-1}\right)\right)$. If $P, Q \in \mathbb{C}[T]$ are nonzero polynomials, $(P, Q)=1$, satisfying the inequality

$$
\left|\alpha-\frac{P}{Q}\right|<\frac{1}{|Q|^{2}},
$$

then there exists $n \geqslant 0$ such that

$$
\frac{P}{Q}=\frac{P_{n}}{Q_{n}},
$$

where $P_{n} / Q_{n}$ denotes the $n$th convergent of $\alpha$.

Proof. This result can be found in [16].

For each $\alpha \in \mathbb{C}\left(\left(T^{-1}\right)\right)$ we write $\lfloor\alpha\rfloor$ for the polynomial part of $\alpha$ and $\{\alpha\}=\alpha-\lfloor\alpha\rfloor$ for the fractional part of $\alpha$, i.e. for

$$
\alpha=\sum_{i=n}^{\infty} a_{i} T^{-i} \quad \text { with } a_{i} \in \mathbb{C},
$$

the polynomial part and the fractional part are

$$
\lfloor\alpha\rfloor=\sum_{i=n}^{0} a_{i} T^{-i}, \quad\{\alpha\}=\sum_{i=1}^{\infty} a_{i} T^{-i},
$$

respectively. The above algorithms for obtaining the partial quotients can be reformulated in $A_{0}=\lfloor\alpha\rfloor, B_{0}=\{\alpha\}$ and $A_{n}=\left\lfloor B_{n-1}^{-1}\right\rfloor, B_{n}=\left\{B_{n-1}^{-1}\right\}$ for all $n \geqslant 1$.

An infinite continued fraction $\left[A_{0}, A_{1}, \ldots\right]$ is called periodic if there are integers $k \geqslant 0$, $\ell \geqslant 1$ such that $A_{n+\ell}=A_{n}$ for all $n \geqslant k$. Thus a periodic continued fraction can be written in the form

$$
\left[A_{0}, A_{1}, \ldots, A_{k-1}, \overline{A_{k}, A_{k+1}, \ldots, A_{k+\ell-1}}\right],
$$

where the bar over $A_{k}, A_{k+1}, \ldots, A_{k+\ell-1}$ indicates that this block of polynomials is repeated indefinitely.

In the classical theory of continued fraction expansions exactly the quadratic irrationals have a periodic expansion. This is not true for the continued fraction algorithm over $\mathbb{C}(T)$. Nevertheless, if we have a periodic expansion then we can calculate it in the usual way, which we will show below.

Let $\alpha \in \mathbb{C}\left(\left(T^{-1}\right)\right)$ be an algebraic element of degree 2 over $\mathbb{C}(T)$, then $\alpha$ is said to be quadratic irrational. In this case $\alpha$ has the form

$$
\alpha=\frac{A+\Delta}{B},
$$

with $A, B \in \mathbb{C}[T], B \neq 0$ and $\Delta^{2}=D \in \mathbb{C}[T]$ and $B \mid\left(D-A^{2}\right)$. A quadratic irrational $\alpha$ must be a root of the equation

$$
X^{2}-\operatorname{Tr}_{\mathbb{C}(T) / \mathbb{C}}(\alpha) X+\mathcal{N}_{\mathbb{C}(T) / \mathbb{C}}(\alpha)=0 .
$$


The other root of (3.3) is the algebraic conjugate of $\alpha$, namely $\alpha^{\prime}=(A-\Delta) / B$. Let us define $\Delta= \pm \sqrt{D}$, where we choose the sign such that

$$
\left|\frac{A-\sqrt{D}}{B}\right| \leqslant\left|\frac{A+\sqrt{D}}{B}\right| \text {. }
$$

Lagrange's Theorem is not true in the form that every quadratic irrational over $\mathbb{C}(T)$ has periodic continued fraction or pseudo-periodic continued fraction (the definition can be found in [19]). But if the quadratic irrational $\alpha=(A+\sqrt{D}) / B$ has a periodic expansion, then this expansion can be obtained using the following algorithm which we will obtain similarly as in the classical theory of continued fraction expansion (see [18, chapter 7.7]). Let $S_{0}=A, T_{0}=B$ and

$$
\alpha_{n}=\frac{S_{n}+\sqrt{D}}{T_{n}}, A_{n}=\left\lfloor\alpha_{n}\right\rfloor, S_{n+1}=A_{n} T_{n}-S_{n}, T_{n+1}=\frac{D-S_{n+1}^{2}}{T_{n}}
$$

for $n \geqslant 0$.

LEMmA 1. Let $\left(\alpha_{n}\right),\left(A_{n}\right),\left(S_{n}\right),\left(T_{n}\right)$ be the sequences defined by (3.4), then $\alpha=$ $\left[A_{0}, A_{1}, \ldots\right]$ is the continued fraction expansion of $\alpha$.

Proof. We have

$$
\alpha_{i}-A_{i}=\frac{S_{i}+\sqrt{D}-A_{i} T_{i}}{T_{i}}=\frac{\sqrt{D}-S_{i+1}}{T_{i}}=\frac{T_{i+1}}{\sqrt{D}+S_{i+1}}=\frac{1}{\alpha_{i+1}}
$$

which verifies that $\alpha=\left[A_{0}, A_{1}, \ldots\right]$. Now we use induction to prove that $S_{i}$ and $T_{i}$ are polynomials such that $T_{i} \neq 0$ and $T_{i} \mid\left(D-S_{i}^{2}\right)$. This holds for $i=0$. If it is true at the $i$ th stage, we observe that $S_{i+1}=A_{i} T_{i}-S_{i}$ is a polynomial. Then the equation

$$
T_{i+1}=\frac{D-S_{i+1}^{2}}{T_{i}}=\frac{D-S_{i}^{2}}{T_{i}}+2 A_{i} S_{i}-A_{i}^{2} T_{i}
$$

establishes that $T_{i+1}$ is also a polynomial. Moreover $T_{i+1}$ cannot be zero, since if it were, we would have $D=S_{i+1}^{2}$, whereas $D$ is not a perfect square. Finally, we have $T_{i}=$ $\left(D-S_{i+1}^{2}\right) / T_{i+1}$, so that $T_{i+1} \mid\left(D-S_{i+1}^{2}\right)$.

From this algorithm it follows that if there are integers $j$ and $k(j<k)$ such that $\left(S_{j}, T_{j}\right)=\left(S_{k}, T_{k}\right)$, then $\alpha_{j+i}=\alpha_{k+i}$ for all $i \geqslant 0$. Hence, $\alpha=(A+\sqrt{D}) / B$ has a periodic expansion and that expansion is of the form

$$
\alpha=\left[A_{0}, \ldots, A_{j-1}, \overline{A_{j}, \ldots, A_{k-1}}\right] .
$$

Moreover, the converse is also true: if the expansion is periodic then there are integers $j<k$ such that $\left(S_{j}, T_{j}\right)=\left(S_{k}, T_{k}\right)$.

In the next lemma we will see how the convergents of the continued fraction expansion of a quadratic irrational $\alpha$ can be used to describe the solutions of a certain Pellian equation. This will be useful in describing all possible right-hand sides of the equation $(1 \cdot 2)$.

LEMMA 2. Let $A B$ be a polynomial which is not a perfect square in $\mathbb{C}[T]$ and let $P_{n} / Q_{n}$ denote the $n$th convergent of the continued fraction expansion of $\sqrt{A B^{-1}}$. Let the sequences $\left(S_{n}\right)$ and $\left(T_{n}\right)$ be defined by (3.4) for the quadratic irrational $\sqrt{A B} / B$. Then

$$
A Q_{n}^{2}-B P_{n}^{2}=(-1)^{n} T_{n+1}
$$


for $n \geqslant 0$.

Proof. Since $\sqrt{A B} \notin \mathbb{C}(T)$, from

$$
\frac{\sqrt{A B}}{B}=\frac{\left(S_{n+1}+\sqrt{A B}\right) P_{n}+T_{n+1} P_{n-1}}{\left(S_{n+1}+\sqrt{A B}\right) Q_{n}+T_{n+1} Q_{n-1}}
$$

it follows that

$$
\begin{aligned}
S_{n+1} Q_{n}+T_{n+1} Q_{n-1} & =B P_{n}, \\
S_{n+1} P_{n}+T_{n+1} P_{n-1} & =A Q_{n} .
\end{aligned}
$$

By combining these two equalities with (3.1), we find (3.5).

In the next section we will apply this lemma in order to reduce the Thue inequality to finitely many Diophantine equations.

\section{From an inequality to a system of equations}

In this section, we will consider the connections between solutions of the equations $(1.3 a),(1.3 b)$ and continued fraction expansion of the corresponding quadratic irrationals in $\mathbb{C}\left(\left(T^{-1}\right)\right)$.

Applying the algorithm for quadratic irrationals from above to

$$
\theta_{1}:=\sqrt{\frac{2 c+1}{2 c}}=\frac{\sqrt{2 c(2 c+1)}}{2 c} \text { and } \theta_{2}:=\sqrt{\frac{c}{c-2}}=\frac{\sqrt{c(c-2)}}{c-2}
$$

we find the following continued fraction expansions:

LEMMA 3. The continued fraction expansion of the quadratic irrationals $\theta_{1}, \theta_{2}$ are given by

$$
\theta_{1}=\left[1,4 c+\frac{1}{2}, \overline{-16 c-4,4 c+1}\right] \quad \text { and } \quad \theta_{2}=\left[1, c-\frac{3}{2}, \overline{-4 c+4, c-1}\right] .
$$

Proof. We prove the statement by applying the above algorithm. For the quadratic irrational $\theta_{1}$ we have $S_{0}=0, T_{0}=2 c$ and therefore

$$
\alpha_{0}=\frac{\sqrt{2 c(2 c+1)}}{2 c}=1+\frac{1}{c}+\mathcal{O}\left(\frac{1}{c^{2}}\right) .
$$

Thus we get $A_{0}=1$. Next we get $S_{1}=1 \cdot 2 c-0=2 c$ and $T_{1}=\left(2 c(2 c+1)-(2 c)^{2}\right) / 2 c=$ 1. It follows that

$$
\alpha_{1}=\frac{2 c+\sqrt{2 c(2 c+1)}}{1}=4 c+\frac{1}{2}-\frac{1}{16 c}+\mathcal{O}\left(\frac{1}{c^{2}}\right),
$$

and therefore $A_{1}=4 c+1 / 2$. Next we get $S_{2}=2 c+1 / 2, T_{2}=-1 / 4$ and

$$
\alpha_{2}=\frac{2 c+\frac{1}{2}+\sqrt{2 c(2 c+1)}}{-\frac{1}{4}}=-16 c-4+\frac{1}{4 c}+\mathcal{O}\left(\frac{1}{c^{2}}\right),
$$

thus $A_{2}=-16 c-4$. Now $S_{3}=2 c+1 / 2, T_{3}=1$ and

$$
\alpha_{3}=\frac{2 c+\frac{1}{2}+\sqrt{2 c(2 c+1)}}{1}=4 c+1-\frac{1}{16 c}+\mathcal{O}\left(\frac{1}{c^{2}}\right),
$$


which implies $A_{3}=4 c+1$. Since in the next step we get $S_{4}=2 c+1 / 2, T_{4}=-1 / 4$, which is the same pair as in step 2, we conclude that we have the periodic expansion as claimed in the statement.

For the second quadratic irrational $\theta_{2}$ we get in the same way with $\left(S_{0}, T_{0}\right)=(0, c-2)$, $\left(S_{1}, T_{1}\right)=(c-2,2),\left(S_{2}, T_{2}\right)=(c-1,-1 / 2),\left(S_{3}, T_{3}\right)=(c-1,2),\left(S_{4}, T_{4}\right)=(c-$ $1,-1 / 2)$, the expansion and therefore the proof is finished.

Assume that $(U, V, Z)$ is a solution of the system $(1 \cdot 3 a)$ and $(1 \cdot 3 b)$. We will show that $V / U$ is a good approximation of $\theta_{1}$ and $U / Z$ is a good rational approximation of $\theta_{2}$ if the absolute value of these fractions is less than 1 . Otherwise the situation is handled in the following lemma.

LEMMA 4. Let $(U, V, Z)$ be a solution of $(1 \cdot 3 a),(1 \cdot 3 b)$, respectively. If $\operatorname{deg} U>\operatorname{deg} V$ or $\operatorname{deg} Z>\operatorname{deg} U$, then all solutions of $(2 \cdot 3)$ have $\operatorname{deg} x=\operatorname{deg} y=0$.

Proof. Indeed, from $\operatorname{deg} V<\operatorname{deg} U$, it follows that $\operatorname{deg}\left(x^{2}+x y-y^{2}\right)<\operatorname{deg}\left(x^{2}+y^{2}\right)$. This implies that $x, y$ are polynomials of the same degree $\operatorname{deg} x=\operatorname{deg} y=k$. Let $\xi_{x}, \xi_{y} \in$ $\mathbb{C}^{\times}$be the leading coefficients of $x, y$, respectively. Since the leading coefficient of $x^{2}+$ $x y-y^{2}$ has to vanish, it follows that $\xi_{x}=((-1 \pm \sqrt{5}) / 2) \xi_{y}$. Now, equation $(1 \cdot 2)$ implies $k=0$ in the following way: Assume that $k>0$. Observe that, since $c$ is not constant and $\operatorname{deg} m \leqslant \operatorname{deg} c$, we have $\operatorname{deg}\left(4 c x^{3} y\right)=\operatorname{deg}\left(6 c x^{2} y^{2}\right)=\operatorname{deg}\left(4 c x y^{3}\right)=\operatorname{deg} c+4 k>$ $\max \left\{\operatorname{deg} m, \operatorname{deg} x^{4}, \operatorname{deg} y^{4}, \operatorname{deg}\left(2 x^{2} y^{2}\right)\right\}$. Therefore, by comparing the leading coefficients in (1.2), we get that

$$
-4 \xi_{x}^{2}+6 \xi_{x} \xi_{y}+4 \xi_{y}^{2}=0
$$

which is in contradiction to the above relation between $\xi_{x}$ and $\xi_{y}$. This contradiction shows that $k=0$.

Now, we discuss the case $\operatorname{deg} U<\operatorname{deg} Z$. We have $\operatorname{deg}\left(x^{2}+y^{2}\right)<\operatorname{deg}\left(-x^{2}+4 x y+y^{2}\right)$, which implies that $x, y$ are polynomials of the same degree $\operatorname{deg} x=\operatorname{deg} y=k$. As above, let $\xi_{x}, \xi_{y} \in \mathbb{C}^{\times}$denote the leading coefficients. Then $\xi_{x}= \pm i \xi_{y}$, and by using equation (4.1) (which was a consequence of (1.2) in the case $k>0$ ), it follows in the same way as above that $k=0$.

So, we are left to consider the cases where $\operatorname{deg} U \leqslant \operatorname{deg} V$ and $\operatorname{deg} Z \leqslant \operatorname{deg} U$ and from now on we will assume that this is the case. We proceed by showing that in this case we have good approximations of $\theta_{1}$ and $\theta_{2}$, respectively.

LEMmA 5. Let $(U, V, Z)$ be a solution of $(1 \cdot 3 a),(1 \cdot 3 b)$, respectively. If $\operatorname{deg} U \leqslant \operatorname{deg} V$, then

$$
\left|\sqrt{\frac{2 c+1}{2 c}}-\frac{V}{U}\right|=\left|\theta_{1}-\frac{V}{U}\right|<\frac{1}{|U|^{2}} .
$$

If $\operatorname{deg} Z \leqslant \operatorname{deg} U$, then

$$
\left|\sqrt{\frac{c}{c-2}}-\frac{U}{Z}\right|=\left|\theta_{2}-\frac{U}{Z}\right|<\frac{1}{|Z|^{2}} .
$$


Proof. The assumption $\operatorname{deg} U \leqslant \operatorname{deg} V$ is equivalent to $|V / U| \geqslant 1$. Moreover, observe that $\left|\theta_{1}\right|=1$. Therefore, we find

$$
\begin{aligned}
\left|\sqrt{\frac{2 c+1}{2 c}}-\frac{V}{U}\right| & =\left|\frac{2 c+1}{2 c}-\frac{V^{2}}{U^{2}}\right| \cdot\left|\sqrt{\frac{2 c+1}{2 c}}+\frac{V}{U}\right|^{-1} \\
& \leqslant \frac{|m|}{|c||U|^{2}}\left(\min \left\{\left|\sqrt{\frac{2 c+1}{2 c}}\right|,\left|\frac{V}{U}\right|\right\}\right)^{-1}=\frac{|m|}{|c||U|^{2}} \leqslant|U|^{-2} .
\end{aligned}
$$

As above from $\operatorname{deg} Z \leqslant \operatorname{deg} U$ it follows $|U / Z| \geqslant 1,\left|\theta_{2}\right|=1$, and consequently we get

$$
\begin{aligned}
\left|\sqrt{\frac{c}{c-2}}-\frac{U}{Z}\right| & =\left|\frac{c}{c-2}-\frac{U^{2}}{Z^{2}}\right| \cdot\left|\sqrt{\frac{c}{c-2}}+\frac{U}{Z}\right|^{-1} \\
& \leqslant \frac{|m|}{|c||Z|^{2}}\left(\min \left\{\left|\sqrt{\frac{c}{c-2}}\right|,\left|\frac{U}{Z}\right|\right\}\right)^{-1}=\frac{|m|}{|c||Z|^{2}} \leqslant|Z|^{-2},
\end{aligned}
$$

which was the claim of the lemma.

We would like to apply Proposition 1 in order to determine all values of $m \in \mathbb{C}[T]$, $\operatorname{deg} m \leqslant \operatorname{deg} c$ for which one of the equations

$$
\begin{aligned}
(2 c+1) U^{2}-2 c V^{2} & =m \\
(c+2) U^{2}-c Z^{2} & =-2 m
\end{aligned}
$$

has a solution. According to Proposition 1 and Lemma 5, for all solutions $(U, V, Z)$ the quotients $V / U, U / Z$ are equal to a convergent of the continued fraction expansion of $\theta_{1}, \theta_{2}$, respectively. For the determination of the corresponding $m$ 's, we use Lemma 2.

Since the period of the continued fraction expansions of $\theta_{1}, \theta_{2}$ are equal to 2 in both cases, according to Lemma 2, we have to consider only the convergents for $n=0, n=1, n=2$. By checking all possibilities, it is now easy to prove the following result.

PROPOSITION 2. Let $m \in \mathbb{C}[T], m \neq 0$ be a polynomial such that $\operatorname{deg} m \leqslant \operatorname{deg} c$. Then:

(i) if equation (1.3a) has a solution in relatively prime polynomials $U$ and $V$ in $\mathbb{C}[T]$ with $\operatorname{deg} U \leqslant \operatorname{deg} V$, then $m=\zeta^{2}, \zeta \in \mathbb{C}^{\times}$and

$$
(U, V) \in\left\{\zeta\left(Q_{2 n}, P_{2 n}\right), 2 \zeta\left(Q_{2 n+1}, P_{2 n+1}\right): n \geqslant 0\right\},
$$

where $P_{n} / Q_{n}$ is a convergent of $\theta_{1}$;

(ii) if equation (1.3b) has a solution in relatively prime polynomials $U$ and $Z$ in $\mathbb{C}[T]$ with $\operatorname{deg} Z \leqslant \operatorname{deg} U$, then $m=\xi^{2}, \xi \in \mathbb{C}^{\times}$and

$$
(U, Z) \in\left\{\xi\left(P_{2 n}^{\prime}, Q_{2 n}^{\prime}\right), 2 \xi\left(P_{2 n+1}^{\prime}, Q_{2 n+1}^{\prime}\right): n \geqslant 0\right\},
$$

where $P_{n}^{\prime} / Q_{n}^{\prime}$ is a convergent of $\theta_{2}$.

Proof. Observe that from the proof of Lemma 3 it follows that $T_{2 n+1}=1$ for $n=0,1, \ldots$ and $T_{2 n}=-1 / 4$ for all $n=1,2, \ldots$, therefore we get the conclusion by Lemma 2 in case (i). We just have to observe that the convergents are only fixed up to a constant $\zeta \in \mathbb{C}$, thus we get the set stated in the lemma.

In the second case we observe that by Lemma 3 we have $T_{2 n+1}=2$ for $n=0,1, \ldots$ and $T_{2 n}=-1 / 2$ for $n=1,2, \ldots$, therefore we get the second assertion (again by Lemma 2 ). 
It follows that we only have solutions if the right-hand side of (1.2) is constant. Moreover, we mention that for $m=0$ there does not exist a solution, since we assume that $c$ is nonconstant.

Now we will discuss the solvability in relatively prime polynomials $U, V$ and $Z$ of the system of equations $(1 \cdot 3 a)$ and $(1 \cdot 3 b)$, where $m \in \mathbb{C}^{\times}$.

We will need the recursive relations for the convergents with odd and even subscripts. First we do it for the convergents of $\theta_{1}$. From $Q_{0}=1, Q_{1}=4 c+1 / 2$ and

$$
\begin{aligned}
Q_{2 n} & =(-16 c-4) Q_{2 n-1}+Q_{2 n-2}, \quad n \geqslant 1, \\
Q_{2 n+1} & =(4 c+1) Q_{2 n}+Q_{2 n-1}, \quad n \geqslant 1,
\end{aligned}
$$

it follows easily that

$$
\begin{aligned}
& Q_{2 n}=-2\left(32 c^{2}+16 c+1\right) Q_{2 n-2}-Q_{2 n-4}, \quad n \geqslant 2, \\
& Q_{2 n+1}=-2\left(32 c^{2}+16 c+1\right) Q_{2 n-1}-Q_{2 n-3}, \quad n \geqslant 2 \text {, }
\end{aligned}
$$

and the analogous relations are valid for $P_{2 n}$ and $P_{2 n+1}$. Now we derive the recursive relations for the convergents of $\theta_{2}$. From $P_{0}^{\prime}=1, P_{1}^{\prime}=c-1 / 2$ and

$$
\begin{aligned}
P_{2 n}^{\prime} & =(-4 c+4) P_{2 n-1}^{\prime}+P_{2 n-2}^{\prime}, \quad n \geqslant 1, \\
P_{2 n+1}^{\prime} & =(c-1) P_{2 n}^{\prime}+P_{2 n-1}^{\prime}, \quad n \geqslant 1,
\end{aligned}
$$

it follows

$$
\begin{aligned}
& P_{2 n}^{\prime}=-2\left(2 c^{2}-4 c+1\right) P_{2 n-2}^{\prime}-P_{2 n-4}^{\prime}, \quad n \geqslant 2, \\
& P_{2 n+1}^{\prime}=-2\left(2 c^{2}-4 c+1\right) P_{2 n-1}^{\prime}-P_{2 n-3}^{\prime}, \quad n \geqslant 2,
\end{aligned}
$$

respectively, and the analogous relations are valid for $Q_{2 n}^{\prime}$ and $Q_{2 n+1}^{\prime}$.

Using the proposition and the recurrences for the convergents we now get the following lemma, which reduces the problem to solve 8 systems of equations which are given by the intersections of the above linear recurrences.

Lemma 6. Let $(U, V, Z) \in \mathbb{C}[T] \times \mathbb{C}[T] \times \mathbb{C}[T]$ be solution of the system of Pellian equations (1.3a) and (1.3b). Then there exist non-negative integers $k$ and $n$ such that $U=$ $\zeta v_{k}$ or $U=2 \zeta v_{k}^{\prime}$ with $m=\zeta^{2}, \zeta \in \mathbb{C}^{\times}$is the solution of one of the following equations

$$
v_{k}= \pm w_{n} \text { or } v_{k}= \pm 2 w_{n}^{\prime} \text { or } 2 v_{k}^{\prime}= \pm w_{n} \text { or } v_{k}^{\prime}= \pm w_{n}^{\prime} \text {, }
$$

where the sequence $\left(v_{k}\right)$ is given by

$$
\begin{aligned}
v_{0} & =1, \quad v_{1}=-\left(64 c^{2}+24 c+1\right), \\
v_{k+2} & =-2\left(32 c^{2}+16 c+1\right) v_{k+1}-v_{k}, \quad \text { for } k \geqslant 0,
\end{aligned}
$$

the sequence $\left(w_{n}\right)$ by

$$
\begin{aligned}
w_{0} & =1, \quad w_{1}=-4 c^{2}+6 c-1, \\
w_{n+2} & =-2\left(2 c^{2}-4 c+1\right) w_{n+1}-w_{n}, \quad \text { for } n \geqslant 0,
\end{aligned}
$$

the sequence $\left(v_{k}^{\prime}\right)$ by

$$
\begin{aligned}
v_{0}^{\prime} & =4 c+\frac{1}{2}, \quad v_{1}^{\prime}=-\frac{1}{2}\left(512 c^{3}+320 c^{2}+48 c+1\right), \\
v_{k+2}^{\prime} & =-2\left(32 c^{2}+16 c+1\right) v_{k+1}^{\prime}-v_{k}^{\prime}, \quad \text { for } k \geqslant 0,
\end{aligned}
$$


and finally the sequence $\left(w_{n}^{\prime}\right)$ is given by

$$
\begin{aligned}
w_{0}^{\prime} & =c-\frac{1}{2}, \quad w_{1}^{\prime}=-\frac{1}{2}\left(8 c^{3}-20 c^{2}+12 c-1\right), \\
w_{n+2}^{\prime} & =-2\left(2 c^{2}-4 c+1\right) w_{n+1}^{\prime}-w_{n}^{\prime}, \quad \text { for } n \geqslant 0 .
\end{aligned}
$$

Proof. If the system of equations $(1.3 a)$ and $(1.3 b)$ has solutions then, by comparing the right-hand sides of the equations $(1 \cdot 3 a)$ and $(1 \cdot 3 b)$, it follows that $\zeta= \pm \xi$ (where $\zeta, \xi$ are the constants in the general form of Proposition 2). Now, we just have to set $v_{k}=Q_{2 k}, v_{k}^{\prime}=$ $Q_{2 k+1}, w_{n}=P_{2 n}^{\prime}$ and $w_{n}^{\prime}=P_{2 n+1}^{\prime}$. Then the assertion about $U$ follows from Proposition 2. The recurrences follow from the above.

Therefore, in order to prove Theorem 1, it suffices to show that $v_{k}=w_{n}$ implies $k=n=$ 0 and the equations $v_{k}=-w_{n}, v_{k}^{\prime}= \pm w_{n}^{\prime}, v_{k}= \pm 2 w_{n}^{\prime}, 2 v_{k}^{\prime}= \pm w_{n}$ have no solutions.

For this purpose we will consider the sequences modulo certain multiples of $c$. This was done earlier in various contexts of systems of Pellian equations (e.g. in $[\mathbf{1}, \mathbf{3}-\mathbf{5}])$.

\section{Congruence relations}

The aim of this section is to find all possible values of $U$ by the so called congruence method introduced first in [5].

LEMma 7. Let the sequences $\left(v_{k}\right),\left(w_{n}\right),\left(v_{k}^{\prime}\right)$ and $\left(w_{n}^{\prime}\right)$ be defined by (4.2), (4.3), (4.4) and (4.5). Then for all $k, n \geqslant 0$ we have

$$
\begin{aligned}
v_{k} & \equiv(-1)^{k}[8 k(2 k+1) c+1] \quad\left(\bmod 64 c^{2}\right), \\
w_{n} & \equiv(-1)^{n-1}[2 n(2 n+1) c-1] \quad\left(\bmod 4 c^{2}\right), \\
v_{k}^{\prime} & \equiv(-1)^{k}\left[4(k+1)(2 k+1) c+\frac{1}{2}\right] \quad\left(\bmod 64 c^{2}\right), \\
w_{n}^{\prime} & \equiv(-1)^{n}\left[(n+1)(2 n+1) c-\frac{1}{2}\right] \quad\left(\bmod 4 c^{2}\right) .
\end{aligned}
$$

Proof. All relations are obviously true for $k, n \in\{0,1\}$. Assume that (5.1) is valid for $k-2$ and $k-1$. Then

$$
\begin{aligned}
v_{k} \equiv & (-2)\left(32 c^{2}+16 c+1\right) v_{k-1}-v_{k-2} \\
\equiv & (-2)(16 c+1)\left[(-1)^{k-1}(8(k-1)(2 k-1) c+1)\right] \\
& -\left[(-1)^{k-2}(8(k-2)(2 k-3) c+1)\right] \\
\equiv & (-1)^{k}[32 c+2 \cdot 8(k-1)(2 k-1) c+2]-(-1)^{k}[8(k-2)(2 k-3) c+1] \\
= & (-1)^{k}[8 k(2 k+1) c+1] \quad\left(\bmod 64 c^{2}\right) .
\end{aligned}
$$

Thus the first congruence follows by induction. Similarly, one can show the other relations for $w_{n}, v_{k}^{\prime}$, and $w_{n}^{\prime}$ by using the recurrence relations (4.3), (4.4) and (4.5), respectively.

By using the congruences from the last lemma we can show that all solutions arising from the equations $v_{k}= \pm w_{n}, v_{k}^{\prime}= \pm w_{n}^{\prime}, v_{k}= \pm 2 w_{n}^{\prime}, 2 v_{k}^{\prime}= \pm w_{n}$ are just the trivial ones.

PROPOSITION 3. If $v_{k}=w_{n}$ then $n=k=0$. The equations $v_{k}=-w_{n}, v_{k}^{\prime}= \pm w_{n}^{\prime}, v_{k}=$ $\pm 2 w_{n}^{\prime}, 2 v_{k}^{\prime}= \pm w_{n}$ have no solutions in non-negative integers $n, k$. 
Proof. Suppose that $k$ and $n$ are non-negative integers such that $v_{k}=w_{n}$. Then, of course, $v_{k} \equiv w_{n}\left(\bmod 4 c^{2}\right)$. By Lemma 7, we have $(-1)^{n} \equiv(-1)^{k} \quad(\bmod 2 c)$ and therefore $n$ and $k$ are both even or both odd. Furthermore, Lemma 7 implies

$$
8 k(2 k+1) c+1 \equiv 1-2 n(2 n+1) c\left(\bmod 4 c^{2}\right)
$$

and

$$
4 k(2 k+1) \equiv-n(2 n+1) \quad(\bmod 2 c) .
$$

From (5.5) we have

$$
4 k(2 k+1)=-n(2 n+1),
$$

which implies $n=k=0$.

Suppose that $k$ and $n$ are non-negative integers such that $v_{k}=-w_{n}$. Then we get in the same way as above that $(-1)^{k}=(-1)^{n-1}$ and therefore $4 k(2 k+1)=-n(2 n+1)$, which implies $n=k=0$ and therefore $-1=1$, a contradiction.

Suppose that $k$ and $n$ are positive integers such that $v_{k}^{\prime}= \pm w_{n}^{\prime}$. Then, of course, $v_{k}^{\prime} \equiv \pm w_{n}^{\prime}$ $\left(\bmod 4 c^{2}\right)$. By Lemma 7, we have $\pm(1 / 2)(-1)^{n-1} \equiv(1 / 2)(-1)^{k}(\bmod c)$. Furthermore, Lemma 7 implies

$$
4(k+1)(2 k+1) c+\frac{1}{2} \equiv-(n+1)(2 n+1) c+\frac{1}{2}\left(\bmod 4 c^{2}\right)
$$

and

$$
4(k+1)(2 k+1) \equiv-(n+1)(2 n+1)(\bmod 2 c) .
$$

From (5.6) we have

$$
4 k(2 k+1)=-n(2 n+1),
$$

which implies $n=k=0$ and therefore $4 c+1 / 2= \pm\left(c-\frac{1}{2}\right)$, a contradiction.

Suppose now that $v_{k}= \pm 2 w_{n}^{\prime}$. Then we get by Lemma 7 that

$$
(-1)^{k}[8 k(2 k+1) c+1] \equiv \pm 2(-1)^{n}\left[(n+1)(2 n+1) c-\frac{1}{2}\right]\left(\bmod 4 c^{2}\right) .
$$

From this we conclude $(-1)^{k} \equiv \pm(-1)^{n-1}(\bmod c)$ and therefore $(-1)^{k}= \pm(-1)^{n-1}$. Consequently, we get $4 k(2 k+1)=-(n+1)(2 n+1)$, a contradiction.

Finally, we consider the case that $k$ and $n$ are positive integers such that $2 v_{k}^{\prime}= \pm w_{n}$. Then Lemma 7 implies that $(-1)^{k}= \pm(-1)^{n}$. Using Lemma 7 once again we get

$$
2\left[4(k+1)(2 k+1) c+\frac{1}{2}\right] \equiv-[2 n(2 n+1) c-1]\left(\bmod 4 c^{2}\right),
$$

which implies $4(k+1)(2 k+1)=-n(2 n+1)$, again a contradiction.

Altogether we have proved the statement of the proposition.

To sum up, we have derived the following result:

CoROllaRY 1. Let $c \in \mathbb{C}[T]$ be a non-constant polynomial, $m \in \mathbb{C}[T]$, deg $m \leqslant$ $\operatorname{deg} c$. If the system of equations $(1.3 a)$ and $(1.3 b)$ has primitive solutions $(U, V, Z)$ with $\operatorname{deg} Z \leqslant \operatorname{deg} U \leqslant \operatorname{deg} V$, then $m=\zeta^{2}$ with $\zeta \in \mathbb{C}^{\times}$and all primitive solutions are given by $(U, V, Z)=( \pm \zeta, \pm \zeta, \pm \zeta)$ with mixed signs.

Proof. This follows directly from Propositions 2 and 3.

In the next section we will use these facts to prove our main theorem. 


\section{Proof of Theorem 1}

Let $(x, y)$ be a solution of the inequality (1.2), and let $U=x^{2}+y^{2}, V=x^{2}+x y-y^{2}$, $Z=-x^{2}+4 x y+y^{2}$. Then $(U, V, Z)$ satisfies the system $(1 \cdot 3 a)$ and $(1 \cdot 3 b)$ for some polynomial $m \in \mathbb{C}[T]$ such that $|m| \leqslant|c|$.

Assume first that $U, V$ and $Z$ (with $\operatorname{deg} Z \leqslant \operatorname{deg} U \leqslant \operatorname{deg} V$ ) are relatively prime. Then Corollary 1 implies that $m=\zeta^{2}$ and in this case we have $(U, V, Z)=( \pm \zeta, \pm \zeta, \pm \zeta)$ with mixed signs and some $\zeta \in \mathbb{C}^{\times}$. Therefore, we have

$$
\begin{aligned}
x^{2}+y^{2} & = \pm \zeta, \\
x^{2}+x y-y^{2} & = \pm \zeta, \\
-x^{2}+4 x y+y^{2} & = \pm \zeta .
\end{aligned}
$$

These equations imply that $\operatorname{deg} x=\operatorname{deg} y$. Let us assume that $\operatorname{deg} x=\operatorname{deg} y=\ell \geqslant 1$ and let the leading coefficient of $x$ be $\xi_{x}$ and the leading coefficient of $y$ be $\xi_{y}$. Then by comparing the leading coefficients in the above equations, we see that $\xi_{x}$ and $\xi_{y}$ have to satisfy the system

$$
\begin{aligned}
\xi_{x}^{2}+\xi_{y}^{2} & =0, \\
\xi_{x}^{2}+\xi_{x} \xi_{y}-\xi_{y}^{2} & =0, \\
-\xi_{x}^{2}+4 \xi_{x} \xi_{y}+\xi_{y}^{2} & =0,
\end{aligned}
$$

which clearly implies $\xi_{x}=\xi_{y}=0$. This gives a contradiction that shows deg $x=\operatorname{deg} y=0$ or equivalently $x, y \in \mathbb{C}$. Clearly, this does not lead to solutions in $(\mathbb{C}[T] \times \mathbb{C}[T]) \backslash \mathbb{C}^{2}$.

Assume now that $d=\operatorname{gcd}(U, V)$ and $\operatorname{deg} d>0$. Let $U=d U_{1}, V=d V_{1}$. Then $U_{1}$ and $V_{1}$ are relatively prime and satisfy

$$
(2 c+1) U_{1}^{2}-2 c V_{1}^{2}=\frac{m}{d^{2}} .
$$

Since $\operatorname{deg}\left(m / d^{2}\right) \leqslant \operatorname{deg} c$, Proposition 2 implies that $m / d^{2}=\zeta^{2}$, i.e. $m=\zeta^{2} d^{2}$. From

$$
4 V^{2}+Z^{2}=5 U^{2}
$$

it follows that $d \mid Z$, say $Z=d Z_{1}$. In that way, we have obtained the primitive triple $\left(U_{1}, V_{1}, Z_{1}\right)$ satisfying

$$
\begin{aligned}
& (2 c+1) U_{1}^{2}-2 c V_{1}^{2}=\zeta^{2}, \\
& (c-2) U_{1}^{2}-c Z_{1}^{2}=-2 \zeta^{2}
\end{aligned}
$$

By Proposition 2, it follows that $\left(U_{1}, V_{1}, Z_{1}\right)=( \pm \zeta, \pm \zeta, \pm \zeta)$ and $(U, V, Z)=$ $( \pm \zeta d, \pm \zeta d, \pm \zeta d)$. Therefore, we have

$$
\begin{aligned}
x^{2}+y^{2} & = \pm \zeta d, \\
x^{2}+x y-y^{2} & = \pm \zeta d, \\
-x^{2}+4 x y+y^{2} & = \pm \zeta d,
\end{aligned}
$$

with mixed signs. Now $(6 \cdot 1 a)$ and $(6 \cdot 1 b)$ imply $y(2 y-x)=0$ or $x(2 y+x)=0$ (depending on the sign) and therefore $(x, y)=(a, 0)$ or $(x, y)=(2 a, a)$ (for the first possible choice of signs in the system above) or $(x, y)=(0, a)$ or $(x, y)=(a,-2 a)$ (for the second possible choice of signs in the system above), where $a \in \mathbb{C}[T]$ with $1<|a| \leqslant|c|^{\frac{1}{4}}$, i.e. $0<\operatorname{deg} a \leqslant 1 / 4 \operatorname{deg} c$. This completes the proof of our main theorem. 
Acknowledgements. This paper was partly written in the frame of a joint AustrianCroatian project granted by the Croatian Ministry of Science, Education and Sports and the Austrian Exchange Service (No. 20/2004 and 23/2006). Moreover, the first author was supported by the Austrian Science Foundation FWF, grants J2407-N12 and NFN S9611 and the second author by the Ministry of Science, Education and Sports, Republic of Croatia, grant 0037110.

\section{REFERENCES}

[1] A. BAKer and H. DavenPort. The equations $3 x^{2}-2=y^{2}$ and $8 x^{2}-7=z^{2}$. Quart. J. Math. Oxford Ser. (2) 20 (1969), 129-137.7.

[2] A. Dujella. The problem of the extension of a parametric family of Diophantine triples. Publ. Math. Debrecen 51 (1997), 311-322.

[3] A. Dujella and B. JADRIJEvić. A parametric family of quartic Thue equations. Acta Arith. 101(2) (2001), 159-169.

[4] A. Dujella and B. JadriJević. A family of quartic Thue inequalities. Acta Arith. 111(1) (2002), 61-76.

[5] A. Dujella and A. Pethô. Generalization of a theorem of Baker and Davenport. Quart. J. Math. Oxford Ser. (2) 49 (1998), 291-306.

[6] C. FUCHS and V. ZIEGLER. On a family of Thue equations over function fields. Monatsh. Math. 147 (2006), 11-23.

[7] C. FuCHS and V. ZIEgLER. Thomas' family of Thue equations over function fields. Quart. J. Math. (Oxford) 57 (2006), 81-91.

[8] B. P. GILL. An analogue for algebraic functions of the Thue-Siegel theorem. Ann. of Math. (2) 31(2) (1930), 207-218.

[9] C. Heuberger, A. Togbé and V. Ziegler. Automatic solution of families of Thue equations and an example of degree 8. J. Symbolic Computation 38 (2004), 1145-1163.

[10] B. JADRIJEVIĆ and V. ZIEGLER. A system of relative Pellian equations and a family of relative related Thue equations. Int. J. Number Theory 2 (4) (2006), 569-590.

[11] G. LeTtL. Thue equations over algebraic function fields. Acta Arith. 117 (2004), 107-123.

[12] G. LeTTL. Parametrized solutions of Diophantine equations. Math. Slovaca 54 (2004), 465-471.

[13] G. Lettl, A. Pethố and P. Voutier. Simple families of Thue inequalities. Trans. Amer. Math. Soc. 351 (1999), 1871-1894.

[14] R. C. MASON. On Thue's equation over function fields. J. London Math. Soc. (2) 24 (1981), 414-426.

[15] R. C. MASon. Diophantine Equations over Function Fields. London Mathematical Society Lecture Note Series, vol. 96 (Cambridge University Press, 1984).

[16] B. De Mathan. Approximations diophantiennes dans un corps local. Bull. Soc. Math. France, Suppl. Mem. 21 (1970).

[17] M. Mignotte, A. Pethő and F. Lemmermeyer. On the family of Thue equations $x^{3}-(n-$ 1) $x^{2} y-(n+2) x y^{2}-y^{3}=k$. Acta Arith. 76 (1996), 245-269.

[18] I. Niven, H. S. ZuCKerman and H. L. Montgomery. An Introduction to the Theory of Numbers (John Wiley, 1991).

[19] W. M. SchmidT. Thue's equation over function fields. J. Austral. Math. Soc. Ser. A 25(4) (1978), 385-422.

[20] W. M. SCHMidT. Continued fractions and diophantine approximation. Acta Arith. 95(2) (2000), 139166.

[21] E. Thomas. Complete solutions to a family of cubic Diophantine equations. J. Number Theory 34 (1990), 235-250.

[22] A. THUE. Über Annäherungswerte algebraischer Zahlen. J. Reine Angew. Math. 135 (1909), 284-305.

[23] H. Stichtenoth. Algebraic Function Fields and Codes (Springer Verlag, 1993).

[24] N. TzanaKis. Explicit solution of a class of quartic Thue equations. Acta Arith. 64 (1993), 271-283.

[25] I. WAKABAYASHI. On a family of quartic Thue inequalities. J. Number Theory 66 (1997), 70-84.

[26] I. WAKABAYASHI. On a family of quartic Thue inequalities, II. J. Number Theory 80 (2000), 60-88.

[27] I. WAKABAYASHI. Cubic Thue inequalities with negative discriminant. J. Number Theory 97 (2002), 222-251. 